\title{
Wavelet-Domain High Resolution Image Reconstruction
}

\author{
Liwen Dong \\ College of Software Engineering \\ University of Science and Technology Liaoning \\ Anshan 114051, China \\ e-mail: lwin181@sina.com.cn
}

\begin{abstract}
The aliasing due to subsampling and the blur from the finite detector size can decrease quality of the images and make fine details and structures difficult to interpret. High resolution images can be reconstructed from several adjacent frames in a sequence by reconstruction process. This paper describes the high resolution image reconstruction method based on wavelet domain. In this approach both the image sequences and the degradation operator are presented by orthogonal wavelet with compact support. Experimental results demonstrate that the proposed method is effective to improve image details.
\end{abstract}

Keywords-image reconstruction; high resolution; wavelet transform

\section{INTRODUCTION}

During image acquisition, image degradation can be caused by many factors such as sensor noise, camera misfocus, random atmospheric turbulence, relative motion between objects and the camera. The purpose of high resolution image reconstruction is to obtain a new image with a higher resolution using collection of low resolution observations which are often shifted, rotated and noisecontaminated. In many practical situations, degradation image can be modeled as

$$
y=D x+n
$$

Where $\mathrm{D}$ is a block matrix, which represents the shift and rotation operator, the blurring operator and subsampling operator. The shift and rotation is caused by the movement of the instrument, and the blur is caused by the point spread function (PSF) of the imaging system. The subsampling operator models the change in resolution between the observations and the desired high resolution image. The series of observations of the high resolution $\mathrm{x}$ are represented by array $\mathrm{y}$. The noise $\mathrm{n}$ can be modeled as additive white Gaussian [1]. Because the acquisition of the images is a realization of the stochastic process, the exact original image can not be computed from the distortion observations. Therefore, with this model, an improved image which is as close as possible to the original image $\mathrm{x}$ can only be obtained from the degraded version $y$.

In imaging system, due to the number and size limits of detector array, spatial sampling frequency cannot meet the sampling theorem. The aliasing in these observations due to inadequate sampling along with the blur from finite detector size can cause serious artifacts, which decrease the image quality and make it hard to get details and structure of images. By employing sufficient detectors to increase the resolution is not an advisable method. However, many imaging systems typically collect a series of images. The series collected are slightly different observations of the same scene. The adjacent frames contain similar but unique information. Exploiting these slightly different but unique information can increase the resolution of image.

The high-resolution restoration idea was first presented by Tsai and Huang [2].One improved resolution image from several subsampled noise-free versions of it was reconstructed using the frequency domain in their approach. Ur and Gross proposed a spatial domain alternative [3]. Irani and Peleg suggested a different approach based on iterative back projection(IBP) for high resolution reconstruction [4]. Cain et al presented a maximum likelihood(ML) technique using an expectation maximization algorithm for the high resolution image restoration. Projection onto convex sets(POCS) algorithm was suggested by Stark and Oskoui. The POCS algorithm can be better than the IBP but the computational demanding limits its applicability [5]. Schultz and Stevenson presented another approach using MAP estimator with the Huber-Markov Random Field prior [6].

In recent years, wavelet analysis has applications in many areas such as image denoising, image deblurring, and image reconstruction. Wavelets analysis are well-suited for image processing because they can achieve image decorrelation and provide a more general and consistent representation of image sequences. The proposed approach uses wavelet transform combined with Bayesian-based maximum a posterior (MAP) method for high resolution image reconstruction.

\section{RECONSTRUCTION METHOD}

There are many methods for high resolution image reconstruction[7-9]. Among them, projections onto convex sets (POCS), iterative back projection (IBP) and MAP are three of the most successful algorithms in spatial domain, while wavelet transform is very popular in frequency domain.

A. Method of projections onto convex sets (POCS method)

Some prior knowledge such as the impulse response of the imaging system and the statistical properties of additive noise can be regarded as a number of constraints of image reconstruction. Each constraint corresponds to a convex set $\left\{C_{j}\right\}(j=1,2, \ldots, \mathrm{m})$ containing the ideal resolution image. 
The intersection of these convex sets $\left\{\bigcap_{j=1}^{m} C_{j}\right\}$ is called the feasible region of the image reconstruction, and accordingly, any element of that is called a feasible solution.

The so-called convex set projection method is a kind of iterative algorithm given some constraint set $C_{j}$ and their respective projection operator $P_{j}$. A feasible solution can be obtained from a given initial results set out, get feasible domain a feasible solution of .

In the constrained case, the iterative method POCS will produce high quality reconstruction image exploiting lot of prior information with convex sets model. But POCS method requires a large amount of calculation, which limits the its application.

\section{B. Iterative back projection (IBP method)}

Irani and Peleg proposed the IBP high resolution reconstruction method. They give an initial estimation for the high resolution image, and then imitated the imaging process to obtain the temporary measurement results. According to the simulation results they update the temporary results. The update equation in IBP method can be expressed as

$$
\hat{X}_{m+1}=\hat{X}+C \sum_{k=1}^{N} Q_{k}^{T}\left[Y_{k}-D_{k} \hat{X}_{m}\right]
$$

Where $\hat{X}_{m}$ is the estimated result of the desired high resolution image at the mth step, $\mathrm{C}$ is a regularization factor $\mathrm{s}$ $D_{k}$ are matrices of degradation operators, $Q_{k}$ are weight matrices, $Y_{k}$ is the simulated measurement, $\mathrm{N}$ is the number of observation images. IBP high resolution image reconstruction method is one with fast speed of convergence.

\section{Maximum a posteriori probability (MAP) method}

Estimating the original image $x$ from the series of observations from Equation (1) is an ill-posed problem since a number of solutions could satisfy the model constraints. By using Bayesian MAP estimation, this ill-posed problem can be convert to a well-posed one. The conditional probability density function of the original image could be defined as

$$
p(x / y)=\frac{P(y / x) P(x)}{P(y)}
$$

Where $P(x / y)$ represents the conditional probability density function of the original image, while $P(y / x)$ represents the probability of observations under the condition of original image. The a priori probability of the original image and that of observation images are represented by $P(x)$ and $P(y)$. The MAP estimation of the unknown image is done by maximizing $P(x / y)$. This can be considered as the best estimate of the original image. Based on Bayesian rule, maximizing $P(x / y)$ is equivalent to maximizing $P(y / x) P(x)$. Assuming that additive noise, observations and original image are all a zero mean Gaussian process, the Bayesian MAP estimation can be used. Thus the high resolution image reconstruction is achieved by minimization of a cost function.
The minimization of the cost function is a MAP estimate and can be defined as

$$
\hat{x}=\arg \operatorname{Min} C(x)
$$

Where $\hat{x}$ is the estimate of a high resolution image, the cost function $C(x)$ can be derived from equation (1).

\section{Wavelet transform}

In recent years the frequency domain reconstruction method has attracted universal attentions. Because of the more accurate description of imaging process, the frequency domain method should be better than the spatial domain in accuracy and anti-noise ability.

In particular, the wavelet transform is effective for local analysis of nonstationary and transient broadband signal. It provides multi-resolution analysis with a single analysis window [10][11]. It uses narrow window for high frequency analysis, while using wide window for low frequency analysis. Image wavelet decomposition is implemented by two-dimensional discrete wavelet transform.

$$
\begin{aligned}
& A_{l}(i, j)=\sum_{m, n \in Z} \tilde{h}(m) \tilde{h}(n) A_{l-1}(2 i-m, 2 j-n) \\
& D_{l}^{1}(i, j)=\sum_{m, n \in Z} \tilde{h}(m) \tilde{g}(n) A_{l-1}(2 i-m, 2 j-n) \\
& D_{l}^{2}(i, j)=\sum_{m, n \in Z} \tilde{g}(m) \tilde{h}(n) A_{l-1}(2 i-m, 2 j-n) \\
& D_{l}^{3}(i, j)=\sum_{m, n \in Z} \tilde{g}(m) \tilde{g}(n) A_{l-1}(2 i-m, 2 j-n)
\end{aligned}
$$

In the layer $l$, with a low-pass filter $\tilde{h}(m)$ and a high-pass filter $\tilde{g}(m)$, the image is decomposed into multi-resolution wavelet coefficients of four bands, which corresponds to Low-Low frequency $\left(A_{l}(i, j)\right)$, Low-High frequency $\left(D_{l}^{1}(i, j)\right)$, High-Low frequency $\left(D_{l}^{2}(i, j)\right)$, High-High frequency $\left(D_{l}^{3}(i, j)\right)$, respectively. Image reconstruction can be achieved by inverse wavelet transform. Wavelet transform provides a general and consistent scheme for representing the inverse problem at multiple resolutions.

\section{E. Proposed approach}

The proposed approach is a one that combines wavelet transform with Bayesian-based MAP iterative method.

As talked in section $C$, the Bayesian MAP estimation can be used, if we assume that additive noise, observations and original image are all a zero mean Gaussian process. Thus the high resolution image reconstruction is achieved by minimization of a cost function.

One of the most practical ways to minimize the cost function is the gradient descent maximization. The gradient descent method is an iterative algorithm extending the IBP algorithm. It is initialized by using the bilinear interpolation of the first frame. The derivatives $d(\tilde{x})$ are yielded by differentiating with respect to $\tilde{x}$.

$$
d(\tilde{x})=\frac{\partial C(\tilde{x})}{\partial \tilde{x}}
$$

Thus the iterative update estimate of high resolution image can be expressed as 


$$
\hat{\tilde{x}}^{n+1}=\hat{\tilde{x}}^{n}-\varepsilon^{n} d\left(\tilde{x}^{n}\right)
$$

Where $\varepsilon^{n}$ is the step size at $n$th iteration. This proposed method decomposes degradation operator and each observation images by using compactly supported orthogonal wavelet. The orthogonal wavelet transform [12]is implemented in the multi-resolution signal analysis framework, which is based on the scaling functions. Both the scaling functions and the wavelet satisfy the orthogonality. The compactly supported orthogonal wavelets have some advantages for high resolution reconstruction. The scaling function and wavelets have good regularity because its support becomes less compact with decomposing layer increase. Distortion of data will be minimal because of the symmetric to their center. The computing speed is fast by only using the convolution of discrete real number data.

In our approach, the degradation operator and the observations are first decomposed by compactly supported orthogonal wavelet. Then do the estimation iteration according to equations (6) and (7) until a preset threshold is satisfied. By inverse wavelet transform, the high resolution image is reconstructed finally. And we use iterative algorithm to monitor calculation process, restrain noise amplification. The wavelet transform can achieve decorrelation of data and consistent representation of images. All above leads to good reconstruction results.

\section{EXPERIMENT AND CONCLUSION}

In order to show the effectiveness of the proposed method for the high resolution reconstruction, twelve subsampled $64 \times 64$ images were generated using an original $256 \times 256$ image (Fig. 3). These images were shifted, rotated and blurred slightly, and contaminated with additive white Gaussian noise with $\sigma^{2}=0.5$. A sample low-resolution image is displayed in Fig. 1. In the simulation, the compactly supported spline wavelet were exploited for the decomposition and reconstruction of images and degradation operators. The high resolution image reconstructed using the proposed method is displayed in Fig. 2. Clearly our algorithm constructs a new image with higher resolution. With respect to the original subsampled images, the detail of the result image is highly improved. The experimental results demonstrates that under the Bayesian framework compactly supported wavelet transform can do a significant job for high resolution reconstruction.

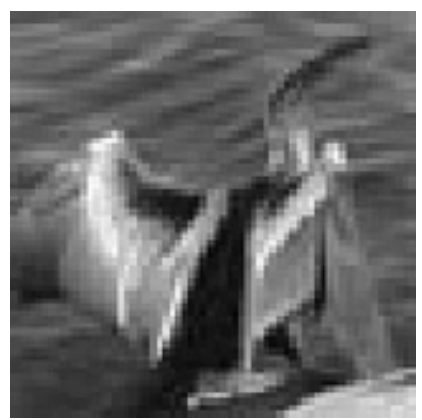

Figure 1. one of 12 subsampled $64 \times 64$ observation images $(64 \times 64)$

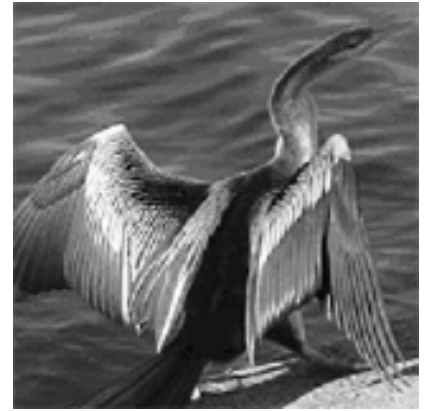

Figure 2. high resolution image reconstructed using the proposed method

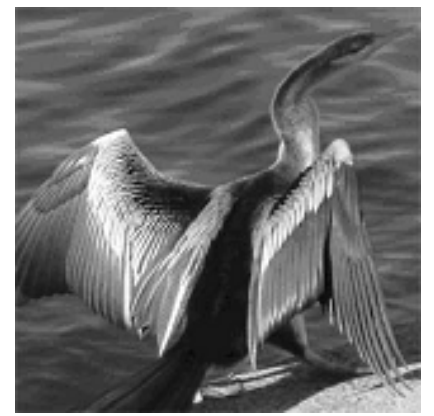

Figure 3. original high resolution $256 \times 256$ image

\section{REFERENCES}

[1] R.C. Gonzalez, R.E. Woods, "Digital Image Processing," Prentice Hall, 2002.

[2] T.S. Huang, R.Y. Tsay, "Multiple frame image restoration and registration," Advances in Computer Vision and Image Processing, vol. 1,pp.317-339, 1984.

[3] H. Ur and D. Gross, "Improved resolution from sub-pixel shifted pictures," CVGIP: Graphical Models and Image Processing, vol.54, pp. 181-186, Mar.1992

[4] Irani M, Peleg S, "Improving resolution by image registration," GVGCP: Graph Models and Image Processing, pp. 231-239, 1991.

[5] Stark. H and Oskoui. P, "High resolution image recovery from imageplane arrays, using convex projections;" J. Opt. Soc. Am. A, Vol.6, pp.1715-1726, 1989.

[6] Schulz. R. R. and Stevenson. R. L, "Extraction of high-resolution frames from video sequences," IEEE Trans. Image Processing, pp.996-1 011, 1996.

[7] Sigeru. Omatu, Hideo. Araki, "Image Restoration by Revised e Bayesian-Based," ADVCOMP :The Fifth International Conference on Advanced Engineering Computing and Applications in Sciences, pp.60-65, 2011.

[8] S Peleg, D. Keren, L Schweitzer, "Improving Image Resolution Using Subpixel Motion," Pattern Recognition Letters, pp.223-226, 1987.

[9] A.M. Tekalp, M. K. Ozkan, M. I. Sezan, "High Resolution Image Reconstruction from Lower-Resolution Image Sequences, and SpaceVarying Image Restoration," Proceedings of IEEE International Conference on Acoustics, Speech and Signal Processing, pp.169-172, 1992.

[10] S.Mallat, "A Theory for Multi-resolution Signal Decomposition: The Wavelet Representation," IEEE Trans. Pattern Anal. and Machine Intell., Vol. 11, no. 7, pp.674-693, 1989.

[11] O. Rioul and M. Vetterli, "Wavelet and signal processing," IEEE Signal Processing Mag., pp. 14-38, 1991.

[12] I. Daubechies, "the orthonomal bases of compactly supported wavelets," Commun Pure Appl Math, pp.909-996,1988. 